ROCZNIKI HUMANISTYCZNE

Tom LXIX, zeszyt $8-2021$

DOI: https://doi.org/10.18290/rh21698-8

ANNA KRZYŻANOWSKA

\title{
À PROPOS DE QUELQUES COMBINAISONS LEXICALES SPÉCIALISÉES DU CHAMP THÉMATIQUE DES NOMS DE MALADIE
}

\author{
ON SOME SPECIALISED LEXICAL COMBINATIONS \\ FROM THE THEMATIC FIELD OF DISEASE NAMES
}

Abstract

The aim of this article is to analyse the syntactic-semantic features of combinations of the NOUN + ADJECTIVE type from French medical vocabulary. Two subsets were distinguished according to the degree of their syntactic and semantic fixedness: recursive structures with a compositional meaning, and structures partially fixed in syntactic and semantic terms. Our research focuses on the description of the relationship between the constitutive nominal element and the adjectival constituent, and on the description of the functioning of selected specialised lexical combinations in press texts where they are sometimes modified. Particular attention was paid to the use of these connections in the metalinguistic function when they appear in a particular context. In the presented analysis of specialised language, the same methodological tools are used as for the analysis of general language.

Keywords: specialised lexical combinations with the nominal component; medical vocabulary; disease names; press text; modification; metalinguistic function.

\section{INTRODUCTION}

Le présent article se propose d'observer quelques structures binaires du champ thématique des noms de maladie, provenant du vocabulaire médical. Sur le plan lexico-sémantique, le micro-ensemble étudié a un caractère hétérogène, car il englobe aussi bien des combinaisons conventionnelles figées

Dr hab. ANNA KRZYŻANOWSKA - Université Marie Curie-Sklodowska, Chaire d'études romanes ; adresse de correspondance : pl. Marii Curie-Skłodowskiej, 4A 20-031 Lublin, Pologne; courriel : anna.krzyzanowska@mail.umcs.pl ; ORCID: https://orcid.org/0000-0001-7155-3612. 
ou semi-figées que d'autres qui ont un sens compositionnel. La description des combinaisons du type NOM+ADJECTIF pose des problèmes du fait que, parfois, il n'est pas facile de déterminer leur degré de figement syntaxique et/ou sémantique. En outre, le constituant nominal, de même que le constituant adjectival peuvent appartenir soit au langage des professionnels, soit à celui des non-spécialistes. En général, les adjectifs servent à spécifier le sémantisme des noms, et à fournir ainsi des sous-classes à partir de l'expansion de leur dénomination.

Notre étude est structurée en deux volets : l'un concernant la description des propriétés linguistiques (syntaxico-sémantiques) de certaines combinaisons lexicales du champ des noms de maladie ; l'autre portant sur le comportement discursif de ces combinaisons et la manière dont elles sont insérées dans un texte journalistique. Notre objectif est de montrer sur quels aspects porte la caractérisation véhiculée par des adjectifs (L'Homme et Zhichao 17).

Pour constituer notre corpus, nous avons exploré les sources d'information suivantes : Le Trésor de la langue française, Le Grand Robert de la langue française (2005), Le nouveau dictionnaire médical (2017) et Le grand dictionnaire terminologique. En dehors des sources dictionnairiques, nous avons eu recours au site web du dictionnaire de l'Académie nationale de médecine, et aussi à celui de l'encyclopédie médicale de Doctissimo. Dans notre recherche, nous avons opté pour un corpus englobant les termes polylexicaux présentant divers niveaux de spécialisation, à savoir : des termes spécialisés, des termes moyennement spécialisés, et aussi des termes non spécialisés (Chebouti 93). Les premiers ont été recensés dans les ouvrages lexicographiques spécialisés (mentionnés plus haut) et les textes écrits rédigés par les chercheurs en médecine, ainsi que dans les articles sur la santé publique (ex. H5N1). Les termes moyennement spécialisés apparaissent dans la presse sur la santé en général (ex. grippe aviaire). En ce qui concerne les termes non spécialisés, ils se rencontrent dans les textes de vulgarisation (ex. grippe du poulet), destinés avant tout au grand public (Delavigne).

La presse électronique d'où sont tirés les exemples pour notre analyse (L'OBS, Le Figaro, Le Quotidien, Slate, Le Monde) comporte 20 articles parus entre février et mai 2009. D'autres articles inclus dans l'analyse (40 au total) ont été sélectionnés de façon non systématique en 2020. 


\section{QUELQUES REMARQUES SUR LE DISCOURS DE VULGARISATION}

L'émergence du journalisme scientifique et le développement des rubriques médicales dans la presse quotidienne, ainsi que la publication des articles à visée pédagogique dans des pages de sites des centres médicaux contribuent largement à la vulgarisation de la terminologie médicale. Dans la rubrique Santé des journaux tels que Le Point, Le Monde, Le Figaro, les journalistes tentent de faire valoir une information scientifique et d'en donner une appréciation. L'utilisation des termes techniques relevant d'un champ d'expérience particulier évite l'ambiguïté typique de la langue courante, mais rend difficile la compréhension des textes pour les non initiés. Selon le point de vue de certains chercheurs, dans la communication médicale médiatisée, la tâche principale du journaliste est de "traduire » ou de reformuler le discours scientifique en discours de semi-vulgarisation scientifique ou discours de vulgarisation (Loffler-Laurian 112, Dechamps 17).

Nous souhaitons préciser que d'une part, le phénomène de la vulgarisation est entendu ici comme un discours " reformulé », et d'autre part, comme " un ensemble de moyens donnés au lecteur de s'approprier les savoirs » (Delavigne, Quand le terme entre 4).

Il est intéressant de rappeler également que, d'après Authier, le mode de fonctionnement du discours de vulgarisation scientifique semble propre, principalement, à instaurer un lien entre l'émetteur et le récepteur. Dans ce cas-là, la fonction dominante de ce type de discours serait « la fonction phatique au sens large et les connaissances scientifiques véhiculées seraient, au moins autant que le but de la communication, des moyens nécessaires à son fonctionnement » (Authier 44).

En partageant ce point de vue, nous tenons également à souligner que le vocabulaire médical se distingue par la prolifération et la stratification de sa terminologie, qui fait qu'on y trouve en même temps des mots de la sphère commune et des termes spécialisés liés au domaine concerné (Vecchiato et Gerolimich 84). Appartenant aussi bien aux professionnels (médecins) qu'aux profanes (patients), ce vocabulaire présente un caractère soit scientifique soit quotidien, en fonction de la situation de la communication (Kacprzak et Goudaillier 1). Pour Delavigne (2013, Quand le terme entre 10), la description des variations dans l'utilisation des termes dans le discours de vulgarisation permet de rendre compte de la réalité des échanges langagiers. La chercheuse souligne également l'importance et le rôle de divers procédés métadiscursifs dont la forte présence est l'une des caractéristiques pertinentes du discours reformulé. 


\section{LES PROPRIÉTÉS SYNTAXICO-SÉMANTIQUES DES COMBINAISONS LEXICALES SPÉCIALISÉES}

Les combinaisons spécialisées faisant partie de notre micro-ensemble correspondent toutes au même modèle syntaxique NOM + ADJECTIF. Ce type de structures semble être plus fréquent et plus productif que celui d'ADJECTIF + NOM ou NOM + DE+ NOM (Vecchiato et Gerolimich 91). Nous accordons une attention particulière aux structures du premier type se laissant regrouper en deux sous-ensembles dont les frontières sont floues. En fait, les termes polylexicaux qui y appartiennent créent un continuum allant de la structure récurrente à sens compositionnel (grippe porcine, maladie professionnelle, idée fixe) au syntagme semi-figé, partiellement compositionnel (fièvre jaune, maladie bleue). Dans notre démarche, nous adoptons un point de vue sémasiologique, sans oublier cependant de rendre compte d'une dimension terminologique des combinaisons étudiées. Pour commencer, nous examinerons de plus près les caractéristiques syntaxico-sémantiques propres à deux sous-ensembles mentionnés plus haut.

\subsection{LES STRUCTURES RECURRENTES A SENS COMPOSITIONNEL}

Les combinaisons qui nous intéressent sont des structures de type binaire à deux constituants entre lesquels il existe un rapport hiérarchique différencié sur le plan syntaxique et sémantique. Le constituant nominal, choisi librement garde son sens habituel, alors que le constituant adjectival avec lequel le mot clé (le nom) se combine de façon privilégiée est sélectionné en fonction de ce dernier. Sur le plan lexico-sémantique, les combinaisons de mots dont il est question peuvent être réparties comme suit :

a) les séries contenant le nom générique maladie:

$\mathrm{N}_{\text {générique }}+\mathrm{Adj}_{\mathrm{rrelationnel}}$ : maladie professionnelle; maladie mentale; maladie acétonémique ;

$\mathrm{N}_{\text {générique }}+\mathrm{Adj}_{\text {qualificatif }}$ : maladie bleue, maladie noire; maladie bronzée ; idée fixe ;

b) les séries où le syntagme lui-même est un nom de maladie :

$\mathrm{N}_{\text {om de maladie }}+\mathrm{Adj}_{\text {relationnel }}$ : grippe aviaire; grippe porcine; grippe intestinale; grippe asiatique, espagnole, russe, mexicaine;

$\mathrm{N}_{\mathrm{om} \mathrm{de} \mathrm{maladie}}+\mathrm{Adj}_{\mathrm{qualificatif}}$ : grippe maligne; fièvre jaune.

Les mots clés tels que maladie (hyperonyme); grippe (hyperonyme) servent à catégoriser des états pathologiques distincts, alors que les constituants adjectivaux jouent leur rôle spécificateur. Prenons comme exemple la structure 
récurrente grippe aviaire. C'est le sémantisme de l'adjectif qui contribue à la spécification notionnelle de la combinaison tout entière, celle-ci renvoyant à la "catégorie des grippes ». La relation entre les deux constituants est donc de l'ordre de la catégorisation.

Au niveau de la composition lexicale, cette structure reste transparente, car ses éléments sont sémantiquement reconnaissables : grippe aviaire renvoie à une grippe «qui se rapporte aux oiseaux, à leur pathologie» (le TLFI). En ce qui concerne le sens terminologique de la combinaison que nous venons d'évoquer, il se rapporte à une "maladie contagieuse due à une Influenza virus touchant les volailles d'élevage et les oiseaux sauvages. Elle peut être transmise à l'homme (Virus H5N1) » (le NDM 391, l'EMD).

Sur le plan syntaxique, grippe aviare, de même que les autres suites nominales comme, par exemple grippe porcine, grippe bovine ou grippe équine, présente un certain degré de cohésion, qui est le garant d'une certaine stabilité structurelle. Ajoutons encore que les constituants adjectivaux aviaire, porcine, bovine, équine ne peuvent pas remplir la fonction prédicative, c'est-à-dire être transformées en structure phrastique de type : sujet + copule + prédicat $(* l a$ grippe est aviaire/porcine/bovine/équine) ou modifiées par un adverbe intensif : *une grippe très aviaire/* très porcine/* très bovine/* très équine (Tutin 57).

Sémantiquement, les combinaisons lexicales dont il est question représentent un contenu lié à un segment thématique particulier. Leur valeur sémantique est déterminée par la position sur ce segment de connaissance et dépend des rapports que les combinaisons étudiées entretiennent avec les autres combinaisons du même segment (Cabré 28).

En ce qui concerne la combinaison de type grippe mexicaine, elle renvoie également à la " catégorie des grippes », à savoir la grippe porcine, définie comme «grippe de type A $(\mathrm{H} 1 \mathrm{~N} 1)$ touchant le porc et pouvant infester l'homme avec transmission interhumaine possible " (le NDM 391, l'EMD). Dans ce cas de figure, il s'agit d'une structure récurrente à sens compositionnel, contenant un adjectif de relation ${ }^{1}$. Elle s'interprète traditionnellement comme un groupe prépositionnel correspondant: l'adjectif mexicain veut dire « originaire du Mexique ».

\footnotetext{
${ }^{1}$ Ajoutons qu'en se fondant sur un corpus médical de langue française, Maniez examine les facteurs déterminant l'emploi de l'adjectif relationnel par opposition à la complémentation par un groupe nominal.
} 


\subsection{LES COMBINAISONS SEMI-FIGEES PARTIELLEMENT COMPOSITIONNELLES}

Les combinaisons de ce type ont un sens partiellement compositionnel : maladie bleue, maladie noire, maladie bronzée, fièvre jaune. La relation entre les deux constituants est de nature hiérarchique. Le choix d'un adjectif s'opère en fonction du mot clé. À la différence des mots clés (maladie; fièvre), les adjectifs ne conservent pas leur sens habituel, mais la motivation sémantique des combinaisons dont ils font partie intégrante reste transparente à travers des hypallages, doublées d'une métonymie.

D'après le TLFI, maladie bleue renvoie à une maladie «caractérisée par une cyanose (coloration bleue de la peau), en rapport avec le passage du sang veineux „bleu” (sang à réoxygéner) dans le sang artériel „rouge” (sang réoxygéné) et un rétrécissement sur la circulation du sang veineux ». Cette définition est proche de celle qu'on trouve dans Le nouveau dictionnaire médical, où maladie bleue désigne des " cardiopathies congénitales se manifestant par une cyanose d'apparition plus ou moins précoce, laquelle est la conséquence de shunts veino-artériels dans le cadre de malformations cardiovasculaires congénitales » (le NDM 528). Le terme polylexical réfère donc à l'un des symptômes visibles au niveau de la peau ou juste une coloration bleue autour des lèvres² ${ }^{2}$.

En ce qui concerne maladie bronzée, cette combinaison (aujourd'hui désuète) renvoie à une " insuffisance surrénale chronique (et) caractérisée par une asthénie profonde, avec hypotension artérielle, à laquelle se joignent des douleurs lombaires, des troubles gastriques, une coloration bronzée de la peau et des taches pigmentaires des muqueuses » (le GDT) ${ }^{3}$. La combinaison mentionnée plus haut réfère au brunissement de la peau analogue au hâle solaire $^{4}$

Les constituants adjectivaux étudiés n'entrent pas non plus dans des structures prédicatives: *la maladie est bleue; * la maladie est noire; * la maladie est bronzée ; * la fièvre est jaune. De plus, aucun élément des suites dont il est question ne peut être remplacé par un autre élément. Au niveau syntagmatique, il y a une altération de la structure de la liaison lexicale établie.

\footnotetext{
${ }^{2}$ https://sante.journaldesfemmes.fr/fiches-sante-du-quotidien/2665443-maladie-bleue-cyanosecongenitale-symptomes-traitement-duree-de-vie/\#: :text=La\%20, consulté le 2.02.2021.

${ }^{3} \mathrm{http} / / /$ gdt.oqlf.gouv.qc.ca/ficheOqlf.aspx?Id_Fiche=8414738, consulté le 9.01.2021

${ }^{4}$ À l'heure actuelle, la combinaison maladie bronzée de la tomate (TSWV) se rapporte à l'une des principales maladies virales des cultures de tomates, dont les symptômes se présentent souvent sous forme de taches sur les feuilles et les tiges.
} 
Idée fixe présente à son tour un cas intéressant qui illustre nettement un décalage entre son emploi courant et son emploi terminologique. Ainsi, en tant que dénomination courante, cette combinaison lexicale a un sens global figuré, en renvoyant soit à « une idée dominante, obsédante, dont l'esprit ne peut se détacher » ou «une obsession, une manie»; soit au «thème, sujet sur lequel on revient sans cesse, par une sorte de manie » (le TLFI $)^{5}$. Compte tenu de ces caractéristiques, il nous semble préférable de ranger cette expression dans la sous-classe des expressions syntaxiquement et sémantiquement semi-figées.

Il importe de signaler que les suites nominales de ce type ont fait la polémique à cause de différentes appellations attribuées à ces expressions polylexicales (Śliwa, « Les unités polylexicales » 409, Lajmi 164, Krzyżanowska 119). Si l'on prend en considération le critère structurel, elles peuvent être traitées comme des noms composés endocentriques (Śliwa, «Les unités polylexicales » 410-413).

En psychologie et psychiatrie, au sens proprement terminologique, idée fixe constitue une structure récurrente à sens compositionnel, même si le concept désigné a une valeur spécialisée. Il convient encore de préciser que cette appellation, selon le point de vue de Jan E. Goldstein, a probablement été utilisée pour la première fois comme un terme médical en 1812 afin de désigner « un lien avec la monomanie ». Il se rapportait à « une simple pathologie mentale », qui se distingue de la « monomanie ${ }^{6} »$.

\section{LE FONCTIONNEMENT DISCURSIF DES COMBINAISONS LEXICALES MOYENNEMENT SPÉCIALISÉES}

Selon M.T. Cabré (26), les unités qui ont un caractère terminologique actualisent un sens spécialisé ou général selon les conditions pragmatiques d'usage en discours. En nous inspirant de cette hypothèse, nous proposons d'observer le comportement des combinaisons lexicales moyennement spécialisées dans les textes de presse. Les combinaisons étudiées y apparaissent soit sous leur forme canonique, soit elles font l'objet de certaines modifications suite à l'insertion dans un contexte lexical et syntaxique spécifique ou

\footnotetext{
${ }^{5} \mathrm{http}$ //stella.atilf.fr/Dendien/scripts/tlfiv5/visusel.exe?12;s=1938605115;r=1;nat=;sol=1; consulté le 8.12. 2020.

${ }^{6} \mathrm{https}$ ://fr.wikipedia.org/wiki/Id\%C3\%A9e_fixe ; Voir aussi Dictionnaire de la Psychiatrie des editions du CILF : www.cilf.fr, consulté le 10.02.2021.
} 
une altération de la structure de la liaison lexicale établie. Dans un premier temps, nous signalons l'emploi canonique de ce type d'expressions, dans un deuxième temps, nous nous concentrons sur les mécanismes de leur manipulation. Lors de notre analyse, nous appliquons au langage spécialisé les mêmes outils méthodologiques que ceux utilisés pour la langue générale (Lerat 24, Śliwa, « Termin » 213).

Les procédés de manipulation mis en œuvre par les journalistes visent le plus souvent à briser un enchaînement attesté, ce qui a pour effet de «désolidariser des éléments d'ordinaire soudés les uns aux autres » (Wozniak 190).

L'analyse du comportement discursif des combinaisons étudiées s'articule autour des trois volets suivants :

- l'emploi canonique

- l'emploi détourné

- l'emploi métalinguistique (Mejri 162, Krzyżanowska, « Variations » 199).

Dans ce qui suit, nous allons essayer de signaler quelques caractéristiques linguistiques de ces trois emplois.

\subsection{L'EMPLOI CANONIQUE}

Le choix de la forme canonique d'une combinaison lexicale donnée n'est pas toujours évident. Certains chercheurs soutiennent que la forme retenue comme forme canonique est celle qui «semble la plus usuelle d'après les dictionnaires actuels » (Klein 38$)^{7}$. Les combinaisons moyennement spécialisées apparaissant dans les articles de presse semi-scientifiques s'actualisent à partir de leur valeur qui renvoie à la " catégorie des grippes ». Elles se caractérisent par la stabilité structurelle (invariabilité des éléments) et sémantique, comme le montre l'exemple ci-dessous :

Landes : un second élevage de canards contaminé par la grippe aviaire

Ce nouveau foyer, identifié dans la commune de Saint-Geours-de-Maremne, a été confirmé par le laboratoire national de référence de l'Anses. [PF1]

\subsection{L'EMPLOI DETOURNE}

Une combinaison moyennement spécialisée peut faire l'objet d'une manœuvre lexicale ou sémantique. Elle subit alors l'opération de détournement, considérée comme un type de manipulation visant à créer un sens discursif

\footnotetext{
${ }^{7}$ C'est la définition qui a été adoptée dans nos recherches.
} 
à partir du sens en langue de la locution originale (Schapira 147). Regardons de plus près quelques exemples.

\subsubsection{Le détournement lexical}

Le procédé dont il est question se manifeste à travers l'insertion d'un élément étranger au niveau de la structure interne du terme polylexical :

À titre d'exemple, sur ce cas de la grippe pas porcine, Alain Joannes réalise un travail très intéressant sur son blog, Journalistiques. [PF 2]

L'élément pas qui porte sur l'adjectif porcine ajoute une valeur négative, en modifiant le sens dénoté (global) de la combinaison utilisée.

Un effet analogue est observé lorsque l'élément inséré est expansé par un autre élément :

La grippe aviaire avait très bien marché. Malheureusement pour la grippe même pas porcine, la sauce a du mal à prendre. Alors que la première nous avait occupés plusieurs semaines, avec sa litanie de cas déclarés, au bout d'une seule semaine, on commencerait presque à ne plus avoir peur de cette variante. [PF 3]

Suite à la modification effectuée, le sens global de la combinaison étudiée est clairement différent de celui de grippe porcine. Il s'agit ici d'un effet de style. Même, adverbe à valeur renchérissante sert à mettre en relief l'adjectif, et aussi à contraster un fait avec un autre.

\subsection{L'EMPLOI METALINGUISTIQUE}

Selon Delavigne (Quand le terme entre 11), les termes ont un statut particulier dans le discours de vulgarisation qu'il faut expliciter. Elle soutient également que ce type de discours met le doigt sur des "mots spécialisés » que les co-énonciateurs sont intuitivement capables d'identifier (Le double jeu 9).

Dans l'exemple ci-dessous, le journaliste s'attend à ce que le récepteur retrouve la forme originale d'un terme polylexical à partir des constituants adjectivaux évoqués dans le titre de l'article :

«Porcine», «mexicaine » ou « américaine»?

La qualification d'un mal contagieux a des conséquences dont on mesure souvent peu la portée.

[PF 4] 
Les constituants adjectivaux guillemetés sont aussi la trace d'une opération métalinguistique locale permettant un certain jeu de mise à distance par rapport à ce qui a été avancé (Komur 72, cf. aussi Stikić 5-6).

Il est intéressant de noter que le fait de recourir à l'opération métalinguistique ne peut avoir pour effet qu'un jeu à vocation esthétique :

La grippe H1N1 est-elle « porcine » ou « mexicaine»?

Quel nom la grippe H1N1 doit-elle porter? Depuis mardi, on l'entend moins sévir sous le nom porcine, elle est désormais devenue «mexicaine». L'allusion au cochon n'a pas seulement ouvert la voie à des calembours parfois douteux, elle a soulevé un débat de fond. La France n'a pas tranché sa position : le ministère de la Santé sur son site parle d'épidémie d'infections respiratoires sévères' ou H1N1. [PF 5]

Le journaliste-énonciateur se préoccupe non du signifié des signes linguistiques, mais il veut mettre l'accent sur un acte linguistique, c'est-à-dire la façon dont on attribue un nom à un phénomène donné. En s'interrogeant sur la validation ou non de la relation prédicative, il fait comme s'il jugeait nécessaire de vérifier s'il utilise bien le code.

Le même cas de figure se présente, lorsque le terme polylexical reçoit les guillemets en bloc. Le signe typographique indique alors que le terme polylexical n'a pas sa signification habituelle, mais qu'il acquiert une valeur métalinguistique. Il s'agit ici d'un jeu créatif, dont le but est d'obtenir un effet stylistique nouveau :

La « vache folle » n'affole pas le Luxembourg

Au Grand-Duché, l'administration des services vétérinaires du ministère de l'Agriculture ne s'inquiète: 'C'est un cas détecté en France et qui, de plus, est limité au niveau de l'exploitation'. [PF 6]

Dans l'exemple suivant, il s'agit avant tout d'une opération métalinguistique de suspension de prise en charge du mot (en l'occurrence, l'adjectif guillemeté) par le locuteur :

Sur nos sites d'information, il faut de la bonne volonté pour trouver les informations rassurantes. Par exemple sur le fait que la grippe n'était pas si «porcine», ou qu'il n'y avait aucun mort aux États-Unis malgré le nombre de cas suspectés... [PF 7]

Si l'adjectif n'avait pas été guillemeté, il aurait pu entrer dans une structure prédicative, en étant en même temps modifié par si «intensif». L'utilisation du signe typographique met en cause cette interprétation. 
On voit que même cette brève analyse de l'activité métalinguistique permet de mettre en évidence la façon dont les termes polylexicaux sont « commentés, recatégorisés, validés, évalués » (Delavigne, De l'(in) constance 15). Les guillemets y apparaissent en tant que moyen opérationnel permettant la mise en exergue d'un mot (ou groupe de mots) donné.

\section{CONCLUSION}

Au terme de cette étude, nous espérons avoir jeté un peu de lumière sur la spécificité des combinaisons lexicales spécialisées du type NOM + ADJECTIF, et aussi sur leur fonctionnement dans les textes de presse. En tant qu'unités complexes, elles ont un caractère hétérogène du point de vue linguistique et terminologique. L'analyse effectuée montre que les combinaisons lexicales moyennement spécialisées participent aux modifications et aux jeux créatifs conformément aux conventions du discours journalistique. Par recours à divers procédés linguistiques et rhétoriques, le journaliste tente de capter et d'influencer la manière de penser de ses lecteurs. C'est aussi un moyen permettant au journaliste de se distancier et de ne pas prendre en charge le contenu de sa proposition. Il semble que, dans les contextes évoqués, à côté de la valeur nominative et de la valeur cognitive véhiculée, les combinaisons étudiées servent à exprimer des fonctions telles que la fonction persuasive, la fonction métalinguistique ou esthétique.

\section{BIBLIOGRAPHIE}

\section{OUVRAGES ET ARTICLES}

Authier, Jacqueline. "La mise en scène de la communication dans des discours de vulgarisation scientifique $»$. Langue française, $n^{\circ} 53,1982$, pp. 34-47.

Cabré, Maria Teresa. «Sur la représentation mentale des concepts : bases pour une tentative de modélisation ». Le sens en terminologie, dir. Henri Béjoint, Philippe Thoiron, Presses Universitaires de Lyon, 2008, pp. 20-38.

Chebouti, Karim. «Le vocabulaire médical du point de vue des trois fonctions primaires », soumis le 24.11.2015, tel.archives-ouvertes.fr/tel-01233280. Consultée le 8.02.2021.

Dechamps, Christina. « Corpus et étude des collocations verbales ». Polissema Revista de Letras do ISCA, $\mathrm{n}^{\circ} 15,2015$, pp. 13-30.

Delavigne, Valérie. De l'(in) constance du métalinguistique dans un corpus de vulgarisation médicale, soumis le 4.06. 2020, journals.openedition.org/corela/11031. Consultée le 18.02.2021. 
Delavigne, Valérie. Le double jeu de l'autonymie. Le fait autonymique dans les langues et les discours, soumis le 17.12.2013, hal.archives-ouvertes.fr/hal-00919527. Consultée le 23.02.2021.

Delavigne, Valérie. Quand le terme entre en vulgarisation, soumis le 18.12.2013; hal.archivesouvertes.fr/hal-00920636. Consultée le 24.02.2021.

Kacprzak, Alicja, Jean-Pierre Goudaillier. «Dénomination des maladies en langue populaire et argotique (de la 'synonymite' des noms de maladie) ». E-ScriptaRomanica, $\mathrm{n}^{\circ}$ 1, 2014, pp. 1-8.

Klein, Jean-René. « Portrait d'un dictionnaire automatique et philologique des proverbes français ». Paremia, $\mathrm{n}^{\circ}$ 26, 2017, pp. 35-40.

Komur, Greta. «Que se cache-t-il sous les guillemets dans la presse écrite française? » Synergies Pologne, $\mathrm{n}^{\circ}$ 6, 2009, pp. 69-78.

Krzyżanowska, Anna. «Étude comparée de la terminologie linguistique : le cas de la phraséologie française et polonaise ». Roczniki Humanistyczne, vol. 65, no 8, 2017, pp. 117-129.

Krzyżanowska, Anna. «Variations, adaptations et modifications des séquences figées ». Neophilologica, $\mathrm{n}^{\circ} 31,2019$, pp. 198-213.

L’Homme, Marie-Claude, Jia Zhichao. «Classement des combinaisons lexicales spécialisées à base nominale dans un dictionnaire d'informatique ». Cahiers de lexicologie, n 106, 2015, pp. 229-251.

Lajmi, Dhouha. "L'actualisation des locutions nominales du discours juridique ». Les locutions nominales en langue générale, éds. Xavier Blanco, Sandrine Fuentes, Salah Mejri, Universitat Autònoma de Barcelona, Servei de Publicacions, 2012, pp. 63-177.

Lerat, Pierre. Les langues spécialisées. PUF, 1995.

Loffler-Laurian, Anne-Marie. "Vulgarisation scientifique : formulation, reformulation, traduction». Langue française, $\mathrm{n}^{\circ}$ 64, 1984, pp. 109-125.

Maniez, François. «L'adjectif dénominal en langue de spécialité : étude du domaine de la médecine». Revue Française de Linguistique Appliquée, nº 14-2, 2009, pp. 117-130.

Mejri, Salah. «Figement, défigement et traduction. Problématique théorique ». Figement, défigement et Traduction. Rencontres méditerranéennes, éds. Pedro Morgorrón Huerta et Salah Mejri, Universidad de Alicante, 2009, pp. 153-163.

Schapira, Charlotte. Les stéréotypes en français. Les proverbes et autres formules. Ophrys, 1999.

Śliwa, Dorota. «Les unités polylexicales dans le cadre du syntagme nominal». Verbum Analecta Neolatina, n 8-2, 2006, pp. 407-415.

Śliwa, Dorota. «Termin i jego relacje w ujęciu poznawczym ». Orbis Linguarum, n 49, 2018, pp. 205-216.

Stikić, Biljana. «Le discours scientifique des médecins spécialistes dans le contexte du Covid-19 : phénomène de transferts sémantiques en français », soumis le 1.12.2020, www.academia.edu/ 43108620/Le_discours_scientifique_des_m\%C3\%A9decins_sp\%C3\%A9cialistes_dans_le_cont exte du Covid $19 \mathrm{ph} \% \mathrm{C} 3 \% \mathrm{~A} 9$ nom $\% \mathrm{C} 3 \% \mathrm{~A} 8$ ne de transferts $\mathrm{s} \% \mathrm{C} 3 \% \mathrm{~A} 9$ mantiques en fran $\%$ C3\%A7 7 ais_\%C3\%A9crit_Academia_edu_2020/1-12. Consultée le 26.02.2021.

Tutin, Agnès. «Les collocations lexicales : une relation essentiellement binaire définie par la relation prédicat-argument ». Langages, $\mathrm{n}^{\circ} 189,2013$, pp. 47-64.

Vecchiato, Sara, Sonia Gerolimich. «La langue médicale est-elle 'trop complexe?' ». Nouvelles Perspectives en Sciences Sociales, $\mathrm{n}^{\circ}$ 9-1, 2013, pp. 81-122.

Wozniak, Audrey. «Le proverbe détourné: étude théorique appliquée à un corpus bilingue francoespagnol ». Paremia, n 18, 2009, pp. 185-196. 


\section{DICTIONNAIRES}

Le Dictionnaire de l'Académie National de Médecine en ligne (le DANM), dictionnaire.academiemedecine.fr/index.php. Consultée le 4.02.2021.

Le grand dictionnaire terminologique (le GDT), gdt.oqlf.gouv.qc.ca/. Consultée le 26.01.2021.

Le Trésor de la langue française informatisé_(le TLFI), atilf.atilf.fr/. Consultée le 3.02.2021.

L'encyclopédie médicale de Doctissimo (l'EMD), www.doctissimo.fr/html/sante/encyclopedie/ encyclopedie medicale.htm. Consultée le 5.02.2021.

Marroun, Ibrahim, Thomas Sené, Jacques Quevaulliers et Abe Fingerhut. Le nouveau dictionnaire médical (le NDM), Elsevier Masson, 2017.

TEXTES DE PRESSE

PF1 : www.lemonde.fr/economie/article/2020/12/10/landes-un-deuxieme-elevage-de-canards-contaminepar-la-grippe-aviaire_6062848_3234.html. Consultée le 8.01.2021.

PF : www.nouvelobs.com/rue89/rue89-nos-vies-connectees/20090504.RUE0109/grippe-pourquoi-1information-s-est-elle-emballee.html. Consultée le 9.01.2021.

PF3 : www.nouvelobs.com/rue89/rue89-nos-vies-connectees/20090504.RUE0109/grippe-pourquoi1-information-s-est-elle-emballee.html. Consultée le 8.01.2021.

PF4 : www.slate.fr/story/4543/nouvelle-grippe-porcine-mexicaine-ou-americaine. Consultée le 10.01.2021.

PF5 : www.lesinrocks.com/2009/04/30/actualite/societe/la-grippe-hln1-est-elle-porcine-ou-mexicaine. Consultée le 11.01.2021.

PF6 : lequotidien.lu/politique-societe/la-vache-folle-naffole-pas-le-luxembourg/. Consultée le 14.01.2021.

PF7 : www.nouvelobs.com/rue89/rue89-nos-vies-connectees/20090504.RUE0109/grippe-pourquoi1-information-s-est-elle-emballee.html. Consultée le 12.01.2021.

\section{À PROPOS DE QUELQUES COMBINAISONS LEXICALES SPÉCIALISÉES DU CHAMP THÉMATIQUE DES NOMS DE MALADIE}

$$
\text { Ré su mé }
$$

Le présent article propose d'étudier les propriétés syntaxico-sémantiques des combinaisons spécialisées de type NOM + ADJECTIF, provenant du vocabulaire médical. Deux sous-ensembles ont été dégagés en fonction de leur degré de figement syntaxique et sémantique : des structures récurrentes à sens compositionnel, et aussi des structures semi-figées partiellement compositionnelles. Les enjeux de notre recherche s'articulent autour de l'interrelation entre le mot clé (le nom) et le constituant adjectival. On décrit également le fonctionnement des combinaisons spécialisées dans les textes de presse, où elles apparaissent sous la forme canonique ou altérée. Dans ce contexte, on s'intéresse en particulier à leur emploi dans la fonction métalinguistique. Il importe de souligner que lors de notre analyse, on applique au langage spécialisé les mêmes outils méthodologiques que ceux utilisés pour la langue générale.

Mots clés : combinaison lexicale spécialisée à base nominale; vocabulaire médical; noms de maladie; textes de presse; modification; fonction métalinguistique. 


\section{O KILKU SPECJALISTYCZNYCH POŁACZENIACH LEKSYKALNYCH Z POLA TEMATYCZNEGO NAZW CHORÓB}

Streszczenie

Celem niniejszego artykułu jest analiza cech syntaktyczno-semantycznych połączeń typu RZECZOWNIK + PRZYMIOTNIK, należących do francuskiego słownictwa medycznego. Wyróżniono dwa podzbiory ze względu na stopień utrwalenia ich struktury wewnętrznej i znaczenia: struktury rekurencyjne o dosłownym znaczeniu oraz struktury częściowo utrwalone pod względem syntaktycznym i znaczeniowym. Problematyka badań skupia się na opisie relacji między konstytutywnym członem nominalnym a członem przymiotnikowym, a także na opisie funkcjonowania wybranych połączeń w tekstach prasowych, w których mogą być modyfikowane. Szczególną uwagę zwrócono na użycie tych połączeń w funkcji metajęzykowej, realizowanej w odpowiednim kontekście. Przedstawiona analiza języka specjalistycznego wykorzystuje takie same narzędzia metodologiczne, jakie są stosowane do analizy języka ogólnego.

Słowa kluczowe: specjalistyczne połączenia leksykalne z członem nominalnym; słownictwo medyczne; nazwy chorób; teksty prasowe; modyfikacja; funkcja metajęzykowa. 